PROCEEDINGS OF THE

AMERICAN MATHEMATICAL SOCIETY

Volume 135, Number 2, February 2007, Pages 523-529

S 0002-9939(06)08471-1

Article electronically published on August 1, 2006

\title{
SUBORDINATING THE KILLED PROCESS VERSUS KILLING THE SUBORDINATE PROCESS
}

\author{
PING HE AND JIANGANG YING
}

(Communicated by Richard Bradley)

\begin{abstract}
In this paper we shall prove that the process obtained by killing first and subordinating next is a subprocess of the process obtained by subordinating first and killing next.
\end{abstract}

\section{INTRODUCTION}

A subordinator is a Lévy process on positive line. A subordinate process is a time change of a Markov process by an independent subordinator. More precisely, given a Markov process $X=\left(X_{t}\right)$ on $(E, \mathcal{B}(E))$ and a subordinator $S=\left(S_{t}\right)$ independent of $X$, then the process $\left(X_{S_{t}}\right)$ is still a Markov process and is called a subordinate process of $X$ by $S$. Its transition semigroup is given by

$$
\mathbf{P}^{x}\left(X_{S_{t}} \in A\right)=\int_{0}^{\infty} \mathbf{P}^{x}\left(X_{s} \in A\right) \mu_{t}(\mathrm{~d} s),
$$

where $\left(\mu_{t}\right)$ is the convolution semigroup of $S$. On the other hand, killing transform means 'killing' a Markov process at a rate given by a decreasing multiplicative functional. Given a decreasing multiplicative functional $M$ of $X$, we define a transition semigroup by

$$
Q_{t}(x, A)=\mathbf{E}^{x}\left(M_{t} ; X_{t} \in A\right), x \in E, A \in \mathcal{B}(E),
$$

which gives birth to a new Markov process $Y$, called a subprocess of $X$ killed by $M$. The simplest case is 'killing' a process upon leaving a domain. In connection with Dirichlet forms, Theorem 3.5 of [6] gives a clear characterization. If both $X$ and $Y$ are symmetric Markov processes on $E$ and are associated with Dirichlet spaces $\left(\mathcal{E}^{X}, \mathcal{F}^{X}\right)$ and $\left(\mathcal{E}^{Y}, \mathcal{F}^{Y}\right)$, respectively, then $Y$ is a subprocess of $X$ if and only if $\left(\mathcal{E}^{Y}, \mathcal{F}^{Y}\right)$ is strongly subordinated to $\left(\mathcal{E}^{X}, \mathcal{F}^{X}\right)$ in the sense that $(1) \mathcal{F}^{Y}$ is a dense subspace of $\mathcal{F}^{X} ;(2)$ for any non-negative $u, v \in \mathcal{F}^{Y}, \mathcal{E}^{X}(u, v) \leq \mathcal{E}^{Y}(u, v)$.

Both are important transformations in the theory of Markov processes. The question is whether the order of transformation can be switched. For example, suppose that $X$ is an $n$-dimensional Brownian motion, $S$ is a one-side $\alpha$-stable process, $\alpha \in(0,1)$, and $D$ is a domain in $\mathbb{R}^{n}$. Subordinating $X$ by $S$ and killing the latter upon leaving the domain $D$, we obtain a symmetric $2 \alpha$-stable process

Received by the editors April 12, 2005 and, in revised form, August 18, 2005.

2000 Mathematics Subject Classification. Primary 60J45.

Key words and phrases. Dirichlet forms, subprocesses, subordinate.

The research of the second author was supported in part by funding from NSFC No. 10271109.

(C)2006 American Mathematical Society Reverts to public domain 28 years from publication 
restricted on $D$, while taking the reverse order, a subordinate process of the killed Brownian motion. What is the connection between these two processes? In this paper we shall prove that the process obtained by killing first and subordinating next is a subprocess of the process obtained by subordinating first and killing next. The problem was raised by Professor P. Fitzsimmons in a private communication, however the real motivation may be found in a recent work of Chen and Song [1, where they use this order to estimate the eigenvalues of $\alpha$-stable processes restricted on a domain.

\section{Main Results}

In this paper we shall exclusively consider symmetric Markov processes and use the Dirichlet form approach. Given an $m$-symmetric Borel right Markov process $X$ on a Lusin state space $E$ with transition semigroup $\left(p_{t}\right)$, there is always a Dirichlet form $(\mathcal{E}, \mathcal{F})$ associated, where

$$
\begin{aligned}
\mathcal{F} & =\left\{u \in L^{2}(E, m): \sup _{t>0} \frac{1}{t}\left(u-p_{t} u, u\right)<\infty\right\} \\
\mathcal{E}(u, u) & =\sup _{t>0} \frac{1}{t}\left(u-p_{t} u, u\right) .
\end{aligned}
$$

We refer to 2 for basic notions in the theory of Dirichlet forms. Note that elements in Dirichlet space always assume their quasi-continuous versions. We prepare two preliminary lemmas first. Assume that $(\mathcal{E}, \mathcal{F})$ is a Dirichlet form on $L^{2}(E, m), \mu$ is its jumping measure, and $\mu^{\prime}$ is another symmetric measure on $E \times E$ dominated by $\mu$. Let $\bar{\mu}^{\prime}(\mathrm{d} x):=\mu^{\prime}(\mathrm{d} x \times E)$. Define

$$
\mathcal{E}^{\prime}(u, v)=\mathcal{E}(u, v)+\mu^{\prime}(u \otimes v)
$$

for $u, v \in D\left(\mathcal{E}^{\prime}\right) \subset \mathcal{F} \cap L^{2}\left(E, \bar{\mu}^{\prime}\right)$, where $u \otimes v(x, y):=u(x) v(y)$. The form $\left(\mathcal{E}^{\prime}, D\left(\mathcal{E}^{\prime}\right)\right)$ is well defined since

$$
\left|\mu^{\prime}(u \otimes v)\right| \leq \frac{1}{2}\left(\bar{\mu}^{\prime}\left(u^{2}+v^{2}\right)\right) .
$$

Lemma 2.1. Let $\mathcal{E}$ and $\mathcal{E}^{\prime}$ be as above. Then it holds that for $u \in D\left(\mathcal{E}^{\prime}\right)$,

$$
\frac{1}{8}\left(\mathcal{E}(u, u)+\bar{\mu}^{\prime}\left(u^{2}\right)\right) \leq \mathcal{E}^{\prime}(u, u) \leq \mathcal{E}(u, u)+\bar{\mu}^{\prime}\left(u^{2}\right)
$$

and

$$
\mathcal{E}^{\prime}(u, u) \geq \frac{1}{2} \mathcal{E}(u, u)
$$

Proof. We shall use a trick which appeared in [7]. The form $(\mathcal{E}, \mathcal{F})$ has the BeurlingDeny decomposition (see e.g. Theorem 3.2.1 of [2])

$$
\mathcal{E}(u, u)=\mathcal{E}^{c}(u, u)+\frac{1}{2} \int_{E \times E}(u(x)-u(y))^{2} \mu(\mathrm{d} x \mathrm{~d} y), u \in \mathcal{F},
$$

where $\mathcal{E}^{c}$ is the local part. Then it is easy to see that for $u \in \mathcal{F} \cap L^{2}\left(E, \bar{\mu}^{\prime}\right)$,

$$
\begin{aligned}
\mathcal{E}^{\prime}(u, u) & =\mathcal{E}^{c}(u, u)+\frac{1}{2} \int_{E \times E}(u(x)-u(y))^{2}\left(\mu-\mu^{\prime}\right)(\mathrm{d} x \mathrm{~d} y)+\bar{\mu}^{\prime}\left(u^{2}\right) \\
& \leq \mathcal{E}(u, u)+\bar{\mu}^{\prime}\left(u^{2}\right)
\end{aligned}
$$


For the other inequality we need to distinguish two cases. Take any constant $a>1$. If

$$
a \cdot \frac{1}{2} \int_{E \times E}(u(x)-u(y))^{2}\left(\mu-\mu^{\prime}\right)(\mathrm{d} x \mathrm{~d} y) \geq \frac{1}{2} \int_{E \times E}(u(x)-u(y))^{2} \mu(\mathrm{d} x \mathrm{~d} y),
$$

then it follows that

$$
\mathcal{E}^{\prime}(u, u) \geq \frac{1}{a}\left[\mathcal{E}(u, u)+\bar{\mu}^{\prime}\left(u^{2}\right)\right]
$$

Conversely if

$$
a \cdot \frac{1}{2} \int_{E \times E}(u(x)-u(y))^{2}\left(\mu-\mu^{\prime}\right)(\mathrm{d} x \mathrm{~d} y) \leq \frac{1}{2} \int_{E \times E}(u(x)-u(y))^{2} \mu(\mathrm{d} x \mathrm{~d} y),
$$

then we have

$$
\begin{aligned}
\frac{a-1}{2} \int_{E \times E}(u(x)-u(y))^{2} \mu(\mathrm{d} x \mathrm{~d} y) & \leq a \cdot \frac{1}{2} \int_{E \times E}(u(x)-u(y))^{2} \mu^{\prime}(\mathrm{d} x \mathrm{~d} y) \\
& \leq 2 a \bar{\mu}^{\prime}\left(u^{2}\right),
\end{aligned}
$$

and for any $b \in[0,1]$,

$$
\begin{aligned}
\mathcal{E}^{\prime}(u, u) & \geq \mathcal{E}^{c}(u, u)+\bar{\mu}^{\prime}\left(u^{2}\right) \\
& =\mathcal{E}^{c}(u, u)+b \bar{\mu}^{\prime}\left(u^{2}\right)+(1-b) \bar{\mu}^{\prime}\left(u^{2}\right) \\
& \geq \mathcal{E}^{c}(u, u)+b \cdot \frac{a-1}{4 a} \int_{E \times E}(u(x)-u(y))^{2} \mu(\mathrm{d} x \mathrm{~d} y)+(1-b) \bar{\mu}^{\prime}\left(u^{2}\right) \\
& \geq \frac{b(a-1)}{2 a} \mathcal{E}(u, u)+(1-b) \bar{\mu}^{\prime}\left(u^{2}\right) .
\end{aligned}
$$

For the first inequality let $b=\frac{1}{2}$ and $a=2$, and for the second, let $b=1$ and $a \rightarrow \infty$. The conclusion follows.

We need another lemma.

Lemma 2.2. Let $(\mathcal{E}, \mathcal{F})$ and $(\tilde{\mathcal{E}}, \tilde{\mathcal{F}})$ be two Dirichlet forms on $L^{2}(E, m)$. Assume that $\mathcal{F}$ is a dense subspace in $(\tilde{\mathcal{E}}, \tilde{\mathcal{F}})$. Let $D$ be an open subset of $E$. Then we have

$$
{\overline{\left(\mathcal{F}^{D}\right)}}^{\tilde{\mathcal{E}}_{1}}=\tilde{\mathcal{F}}^{D},
$$

where ${\overline{\left(\mathcal{F}^{D}\right)}}^{\tilde{\mathcal{E}}_{1}}$ is the closure of $\mathcal{F}^{D}$ under the $\tilde{\mathcal{E}}_{1}$-norm.

Proof. Note that $\tilde{\mathcal{F}}=\overline{\mathcal{F}}^{\tilde{\mathcal{E}}_{1}}$. The above result says that the closure and restriction commute.

Set $\mathcal{F}_{0}:={\overline{\left(\mathcal{F}^{D}\right)}}^{\tilde{\mathcal{E}}_{1}}$. Obviously $\mathcal{F}_{0} \subset \tilde{\mathcal{F}}^{D}$. We will first prove that $\mathrm{b} \mathcal{F}_{0}$, the set of bounded functions in $\mathcal{F}_{0}$, is an algebraic ideal of $\mathrm{b} \tilde{\mathcal{F}}$, the set of bounded functions in $\tilde{\mathcal{F}}$. Take a bounded function $u \in \mathcal{F}_{0}$ and a bounded function $v \in \tilde{\mathcal{F}}$. We need to show that $u v \in \mathcal{F}_{0}$. There exists a sequence $u_{n} \in \mathcal{F}^{D}$ and a sequence $v_{n} \in \mathcal{F}$ such that $u_{n} \rightarrow u$ and $v_{n} \rightarrow v$ both in the $\tilde{\mathcal{E}}_{1}$-norm. Clearly $u_{n} v_{n} \in \mathcal{F}^{D}$ since $\mathrm{b} \mathcal{F}^{D}$ is an algebraic ideal of $\mathrm{b} \mathcal{F}$.

We may assume without loss of generality that $\left\{u_{n}\right\}$ is uniformly bounded. In fact, assume that $|u| \leq c$ and let $u_{n}^{\prime}:=(-c) \vee u_{n} \wedge c$. Then $\left\{u_{n}^{\prime}\right\} \subset \mathcal{F}^{D}$ is uniformly bounded and converges to $u$ q.e. Furthermore since $\tilde{\mathcal{E}}\left(u_{n}^{\prime}, u_{n}^{\prime}\right) \leq \tilde{\mathcal{E}}\left(u_{n}, u_{n}\right)$, there exists a subsequence whose Cesaro mean, which is still in $\mathcal{F}^{D}$ and uniformly bounded, converges to $u$ in the $\tilde{\mathcal{E}}_{1}$-norm. Similarly $\left\{v_{n}\right\}$ may also be assumed to be uniformly bounded. 
By the Markovian property,

$$
\tilde{\mathcal{E}}\left(u_{n} v_{n}, u_{n} v_{n}\right) \leq\left|v_{n}\right|_{\infty} \cdot \tilde{\mathcal{E}}\left(u_{n}, u_{n}\right)+\left|u_{n}\right|_{\infty} \cdot \tilde{\mathcal{E}}\left(v_{n}, v_{n}\right) .
$$

It follows that $\left\{u_{n} v_{n}\right\}$ is $\tilde{\mathcal{E}}$-bounded. Since $u_{n} v_{n}$ converges to $u v$ q.e., the Cesaro mean of $\left\{u_{n} v_{n}\right\}$ (or a subsequence) converges to $u v$ in the $\mathcal{E}_{1}$-norm. Hence the consequence $u v \in \mathcal{F}_{0}$ follows.

Now by a result of Silverstein in [5], there is a quasi-open set $B$ such that

$$
\mathcal{F}_{0}=\left\{u \in \tilde{\mathcal{F}}:\left.u\right|_{B^{c}}=0 \text { a.e. }\right\} .
$$

Obviously $B \subset D$ q.e. However $\mathcal{F}^{D} \subset \mathcal{F}_{0}$ and thus $D \backslash \bar{B}$ is the quasi-open null set. It implies that $D=B$ q.e. and $\mathcal{F}_{0}=\mathcal{F}^{D}$.

Let $X=\left(X_{t}, \mathbf{P}^{x}\right)$ be a symmetric Markov process on the state space $(E, \mathcal{E})$ and let the transition semigroup $\left(p_{t}\right)$ be associated with the Dirichlet space $(\mathcal{E}, \mathcal{F})$ on $L^{2}(E, m)$. Let $D$ be an open subset of $E$ and $\tau_{D}$ the first exit time of $D$. Denote by $X^{D}$ the subprocess of $X$ killed at leaving $D$ with transition semigroup $\left(p_{t}^{D}\right)$ and Dirichlet space $\left(\mathcal{E}, \mathcal{F}^{D}\right)$, where $\mathcal{F}^{D}$ is the set of elements in $\mathcal{F}$ vanishing on the complement of $D$. We sometimes say that $X^{D}$ or $\mathcal{F}^{D}$ is the restriction of $X$ or $\mathcal{F}$ on $D$. A convolution semigroup $\mu=\left\{\mu_{t}\right\}$ on $(0, \infty)$ is called a subordinator. Its Laplace transform is

$$
\int_{0}^{\infty} e^{-x \xi} \mu_{t}(d \xi)=\exp (-t \phi(x))
$$

where $\phi$ is called the Laplace exponent of $\mu$ and may be represented as

$$
\phi(x)=b x+\int\left(1-e^{-x \xi}\right) n(d \xi),
$$

with a drift $b \geq 0$ and Lévy measure $n$ on $(0, \infty)$ satisfying an integrability condition

$$
\int_{0}^{\infty}(1 \wedge \xi) n(d \xi)<\infty
$$

The subordinator $\mu$ is uniquely determined by $\phi$.

We may now define a new kernel $\left(p_{t}^{\phi}\right)$ as

$$
p_{t}^{\phi}(x, B)=\int_{0}^{\infty} p_{s}(x, B) \mu_{t}(\mathrm{~d} s),
$$

which is a transition semigroup on $E$ and is still $m$-symmetric. Let $X^{\phi}$ be the right Markov process (for the definition, see e.g., 4]) with semigroup $\left(p_{t}^{\phi}\right)$. We usually say that we obtain $X^{\phi}$ through a subordinator $\mu$. We now have two transforms: restriction and subordination. Both make sense for any right Markov process. Let $X^{\phi, D}$ be the process obtained through subordinator $\mu$ first and then killed at leaving $D$ and let $X^{D, \phi}$ be the process obtained by the other way around, i.e., killed at leaving $D$ first and then subordinated.

Theorem 2.1. The process $X^{D, \phi}$ is a subprocess of $X^{\phi, D}$ killed by a decreasing $M F$.

Proof. Let $\left(\mathcal{E}^{D}, \mathcal{F}^{D}\right),\left(\mathcal{E}^{\phi}, \mathcal{F}^{\phi}\right),\left(\mathcal{E}^{D, \phi}, \mathcal{F}^{D, \phi}\right)$ and $\left(\mathcal{E}^{\phi, D}, \mathcal{F}^{\phi, D}\right)$ be the Dirichlet forms corresponding to $X^{D}, X^{\phi}, X^{D, \phi}$ and $X^{\phi, D}$, respectively. Then $\mathcal{F}^{\phi, D}=$ $\left(\mathcal{F}^{\phi}\right)^{D}$, the restriction of $\mathcal{F}^{\phi}$ on $D$, and $\mathcal{E}^{\phi, D}=\mathcal{E}^{\phi}$ on $\mathcal{F}^{\phi, D}$. By a result in 
Theorem 3.5 of [6], we need only show that

(1) $\mathcal{F}^{D, \phi}$ is a dense subspace in $\mathcal{F}^{\phi, D}$ with respect to the $\mathcal{E}_{1}^{\phi, D}$-norm.

(2) For any non-negative functions $u, v \in \mathcal{F}^{D, \phi}$, it holds that

$$
\mathcal{E}^{D, \phi}(u, v) \geq \mathcal{E}^{\phi}(u, v) \text {. }
$$

A result in [3] allows us to write down explicitly the Dirichlet form of subordination. Actually it may be verified by definition that

$$
\begin{aligned}
\mathcal{F}^{\phi} & =\left\{u \in L^{2}(E, m): \int_{0}^{\infty}\left(u-p_{t} u, u\right) n(\mathrm{~d} t)<\infty\right\}, \\
\mathcal{E}^{\phi}(u, u) & =\int_{0}^{\infty}\left(u-P_{t} u, u\right) n(\mathrm{~d} t) .
\end{aligned}
$$

It is easy to check that $\left(u-P_{t} u, u\right) \leq(1 \wedge t) \mathcal{E}_{1}(u, u)$ and then

$$
\mathcal{E}^{\phi}(u, u) \leq \int_{0}^{\infty}(1 \wedge t) n(\mathrm{~d} t) \cdot \mathcal{E}_{1}(u, u) .
$$

Hence $\mathcal{F} \subset \mathcal{F}^{\phi}$. Moreover by 3] $\mathcal{F}$ is dense in $\mathcal{F}^{\phi}$. For $u \in \mathcal{F}^{\phi}$ the form may be written as

$$
\mathcal{E}^{\phi}(u, u)=b \mathcal{E}(u, u)+\frac{1}{2} \int_{E \times E}(u(x)-u(y))^{2} j(x, \mathrm{~d} y) m(\mathrm{~d} x)+\int_{E} u^{2}(x) k(x) m(\mathrm{~d} x),
$$

where

$$
\begin{aligned}
j(x, \mathrm{~d} y) & =\int_{0}^{\infty} p_{t}(x, \mathrm{~d} y) n(\mathrm{~d} t), \\
k(x) & =\int_{0}^{\infty}\left(1-p_{t} 1(x)\right) n(\mathrm{~d} t) .
\end{aligned}
$$

Similarly, $\mathcal{F}^{D}$ is a dense subspace of $\mathcal{F}^{D, \phi}$ and for $u \in \mathcal{F}^{D, \phi}$, we have

$$
\begin{aligned}
\mathcal{E}^{D, \phi}(u, u)= & \int_{0}^{\infty}\left(u-p_{t}^{D} u, u\right) n(\mathrm{~d} t) \\
= & b \mathcal{E}(u, u)+\frac{1}{2} \int_{D \times D}(u(x)-u(y))^{2} j^{D}(x, \mathrm{~d} y) m(\mathrm{~d} x) \\
& +\int_{D} u^{2}(x) k^{D}(x) m(\mathrm{~d} x),
\end{aligned}
$$

where

$$
\begin{aligned}
j^{D}(x, \mathrm{~d} y) & =\int_{0}^{\infty} p_{t}^{D}(x, \mathrm{~d} y) n(\mathrm{~d} t), \\
k^{D}(x) & =\int_{0}^{\infty}\left(1-p_{t}^{D} 1(x)\right) n(\mathrm{~d} t) .
\end{aligned}
$$

It is now easy to verify that for any $u, v \in \mathcal{F}^{D}$,

$$
\mathcal{E}^{D, \phi}(u, v)=\mathcal{E}^{\phi}(u, v)+\int_{D \times D} u(x) v(y) q(x, \mathrm{~d} y) m(\mathrm{~d} x),
$$

where

$$
q(x, \mathrm{~d} y):=\int_{0}^{\infty} \mathbf{P}^{x}\left(X_{t} \in \mathrm{d} y, t \geq \tau_{D}\right) n(\mathrm{~d} t)=\int_{0}^{\infty}\left(p_{t}(x, \mathrm{~d} y)-p_{t}^{D}(x, \mathrm{~d} y)\right) n(\mathrm{~d} t) .
$$


Hence (2) holds. It follows from Lemma 2.1 that the $\mathcal{E}_{1}^{\phi, D}$-norm (or $\mathcal{E}_{1}^{\phi}$-norm) is dominated by the $\mathcal{E}_{1}^{D, \phi}$-norm up to a constant. Thus we have $\mathcal{F}^{D, \phi} \subset \mathcal{F}^{\phi, D}$ and it also implies that $\mathcal{F}^{D, \phi}$ and $\mathcal{F}^{D}$ have the same closure under the $\mathcal{E}_{1}^{\phi}$-norm. Then Lemma 2.2 implies that $\mathcal{F}^{D}$ is dense in $\mathcal{F}^{\phi, D}$ with respect to the $\mathcal{E}_{1}^{\phi}$-norm and the conclusion follows.

Remark. It follows from Lemma 2.1 that the $\mathcal{E}^{\phi}$-norm is dominated by half of the $\mathcal{E}^{D, \phi}$-norm. It is interesting to know if $\frac{1}{2}$ is the best constant since it involves the eigenvalue estimate of $X^{\phi, D}$ in terms of $X^{D, \phi}$. It is shown in 1 that when $\phi$ is a complete Bernstein function it actually holds that $\mathcal{E}^{\phi}(u, u) \leq \mathcal{E}^{D, \phi}(u, u)$.

Killing a process upon leaving a set is a special case of killing a process through a decreasing multiplicative functional. Let us now consider a general decreasing exact multiplicative functional $M=\left(M_{t}\right)$. Denote by $D$ the set of permanent points of $M$, i.e., $D=\left\{x: \mathbf{P}^{x}\left(M_{0}=1\right)=1\right\}$. Let $X^{M}$ be the subprocess of $X$ killed by $M$, with state space $D$ and transition function $\left(p_{t}^{M}\right)$ that is assumed to be also $m$-symmetric. Clearly $p_{t}^{M} f(x)=\mathbf{E}^{x}\left(M_{t} f\left(X_{t}\right)\right) \leq p_{t} f(x)$ for any measurable function $f \geq 0$ and $x \in E$.

Theorem 2.2. The subordinate process $X^{M, \phi}$ of $X^{M}$ by subordinator $\mu$ is a subprocess of the subordinate process $X^{\phi}$ of $X$, killed by a decreasing multiplicative functional with bivariate Revuz measure $q(x, \mathrm{~d} y) m(\mathrm{~d} x)$, where

$$
q(x, \mathrm{~d} y)=\int_{0}^{\infty}\left(p_{t}(x, \mathrm{~d} y)-p_{t}^{M}(x, \mathrm{~d} y)\right) n(\mathrm{~d} t) .
$$

Proof. Let $\left(\mathcal{E}^{M}, \mathcal{F}^{M}\right)$ and $\left(\mathcal{E}^{M, \phi}, \mathcal{F}^{M, \phi}\right)$ be the Dirichlet forms associated with $X^{M}$ and $X^{M, \phi}$, respectively. Again by the characterization result in Theorem 3.5 of 6], we need to check that $(1) \mathcal{F}^{M, \phi} \subset \mathcal{F}^{\phi} ;(2) \mathcal{E}^{M, \phi}(u, v) \geq \mathcal{E}^{\phi}(u, v)$ for any nonnegative functions $u, v \in \mathcal{F}^{M, \phi}$; (3) the closure of $\mathcal{F}^{M, \phi}$ in $\mathcal{F}^{\phi}$, denoted by $\mathcal{F}_{0}$, is equal to $\mathcal{F}^{\phi, D}$. Since $X^{M}$ is a subprocess of $X$, it is known that (i) $\mathcal{F}^{M} \subset \mathcal{F}$; (ii) for non-negative functions $u, v \in \mathcal{F}^{M}, \mathcal{E}^{M}(u, v) \geq \mathcal{E}(u, v)$; (iii) the closure of $\mathcal{F}^{M}$ in $\mathcal{F}$ is equal to $\mathcal{F}^{D}$. It is similar to verify that for any $u, v \in \mathcal{F}^{M}$,

$$
\mathcal{E}^{M, \phi}(u, v)=\mathcal{E}^{\phi}(u, v)+\int_{E \times E} u(x) v(y) q(x, \mathrm{~d} y) m(\mathrm{~d} x),
$$

where

$$
q(x, \mathrm{~d} y):=\int_{0}^{\infty}\left(p_{t}(x, \mathrm{~d} y)-p_{t}^{M}(x, \mathrm{~d} y)\right) n(\mathrm{~d} t)
$$

Thus (2) follows. It follows from Lemma 2.1 again that the $\mathcal{E}_{1}^{\phi}$-norm is dominated by $\mathcal{E}^{M, \phi}$ up to a constant and then it holds that $\mathcal{F}^{M, \phi} \subset \mathcal{F}^{\phi}$. Finally since $\mathcal{F}^{M}$ is dense in $\mathcal{F}^{D}$, which is dense in $\mathcal{F}^{D, \phi}$, it follows that $\mathcal{F}^{M}$ is dense in $\mathcal{F}^{D, \phi}$. However $\mathcal{F}^{D, \phi}$ is dense in $\mathcal{F}^{\phi, D}$ by Theorem 2.1 and $\mathcal{F}^{M} \subset \mathcal{F}^{M, \phi}$. This implies (3). Finally by [7, the bivariate Revuz measure of the multiplicative functional is certainly $q(x, \mathrm{~d} y) m(\mathrm{~d} x)$. This completes the proof.

\section{ACKNOWLEDGEMENTS}

The authors would like to thank Professor Zhenqing Chen for a stimulating discussion. 


\section{REFERENCES}

[1] Chen, Z.-Q., Song, R., Two-sided eigenvalue estimates for subordinate processes in domain, to appear in J. Funct. Anal.

[2] Fukushima, M., Oshima, Y. and Takeda, M., Dirichlet Forms and Symmetric Markov Processes, De Gruyter, Berlin, 1994 MR1303354(96f:60126)

[3] Okura, H., Recurrence and transience criteria for subordinated Markov processes, Forum Math., 14(2002), 121-146 MR.1880197 (2002m:60146)

[4] Sharpe, M.J., General Theory of Markov Processes, Academic Press, 1988 Mr0958914 (89m:60169)

[5] Silverstein, M., Symmetric Markov processes, Lecture notes in Math, Springer, 1974 MR0386032 (52:6891)

[6] Ying, J., Killing and subordination, Proc. Amer. Math. Soc., 124, No. 7(1996), 2215-2222 MR1346994 (97a:31005)

[7] Ying, J., Characterization of bivariate Revuz measures, Kyushu J. Math., 51, No.1(1997), 185-193 MR:1437316 (98f:60148)

Department of Applied Mathematics, Shanghai University of Finance and Economics, Shanghai, People's Republic of China

E-mail address: pinghe@mail.shufe.edu.cn

Institute of Mathematics, Fudan University, Shanghai, People's Republic of China

E-mail address: jgying@fudan.edu.cn 